\title{
PROMIS Adult Mental Health Domain
}

National Cancer Institute

\section{Source}

National Cancer Institute. PROMIS Adult Mental Health Domain. NCI Thesaurus. Code C121994.

A Patient Reported Outcomes Measurement Information System (PROMIS) domain that assesses the status of an individual's mental health. 